\title{
Mechanical circulatory support during high-risk percutaneous coronary intervention in a young male patient
}

\author{
Artur Pawlik¹, Paweł Kleczyński², Dariusz Dudek³, Artur Dziewierz²,3, Stanisław Bartuś̌,3 \\ ${ }^{1}$ Department of Cardiology and Cardiovascular Interventions, University Hospital, Krakow, Poland \\ 2Department of Interventional Cardiology, Jagiellonian University Medical College, John Paul II Hospital, Krakow, Poland \\ ${ }^{3}$ Second Department of Cardiology, Jagiellonian University Medical College, Krakow, Poland
}

Adv Interv Cardiol 2020; 16, 3 (61): 347-348

DOI: https://doi.org/10.5114/aic.2020.99274

A 58-year-old man, with a history of myocardial infarction, hypercholesterolemia, smoking, arterial hypertension, chronic obstructive pulmonary disease, paroxysmal atrial fibrillation, and stroke, was transferred from another hospital to our department to undergo high-risk percutaneous coronary intervention $(\mathrm{PCl})$. Previously performed coronary angiography revealed a multi-vessel disease with significant stenosis of the left main (LM) and left ascending coronary artery (LAD), as well as occlusions of the right coronary artery and circumflex artery (Figure $1 \mathrm{~A}$ ). Due to severely depressed left ventricular (LV) function (ejection fraction of 25\%) and the need for complex $\mathrm{PCl}$ of the last remaining vessel, after the discussion with the heart team, he was scheduled for $\mathrm{PCl}$ with the support of a percutaneous left ventricular assist device (pLVAD) - so-called 'protected PCl' [1].

After the initial peripheral angiography confirming eligibility for using pLVAD, a $14 \mathrm{Fr}$ sheath was inserted into the right femoral artery. The Impella CP system (Abiomed Inc., Danvers MA, USA) was delivered to the LV and started providing a cardiac output of $3.4 \mathrm{l} / \mathrm{min}$. Subsequently, intravascular ultrasound (IVUS) guided PCI using the right transradial approach was started. During each balloon inflation, the curve of blood pressure measured in the ascending aorta was flattened (Figure 1 B), which meant that cardiac output was provided only by pLVAD. Eventually, a $3.5 \times 48 \mathrm{~mm}$ drug-eluting stent was successfully implanted in the LM/LAD (Figures $1 \mathrm{C}, \mathrm{D}$ ). The femoral artery was closed with the Perclose ProGlide closure system (Abbott Cardiovascular, Santa Clara, CA, USA). Further hospitalization was uncomplicated, and after 3 days, the patient was discharged home.

The Impella CP provides up to $4 \mathrm{l} / \mathrm{min}$ cardiac output covering $70-100 \%$ of the heart contractile function [2]. Therefore, the arterial pressure drops during the described procedure did not manifest themselves either as severe cardiac arrhythmias or cardiac arrest. The operator was able to continue the intervention, prepare highly calcified and complex lesions, and apply advanced percutaneous techniques, resulting in good stent apposition. As the introduction of Impella CP is relatively easy and safe, we assume that this device should be used more often in carefully selected patients, especially since the national healthcare system in Poland reimburses its use during high-risk PCls [3]. The heart team consultation should help in choosing patients who will benefit from this technology the most.

\section{Conflict of interest}

The authors declare no conflict of interest.

\section{Corresponding author:}

Artur Pawlik, Department of Cardiology and Cardiovascular Interventions, University Hospital, Krakow, Poland, phone: +48 519614804 , e-mail: arturo.pawlik@gmail.com

Received: 28.03.2020, accepted: 18.04.2020. 

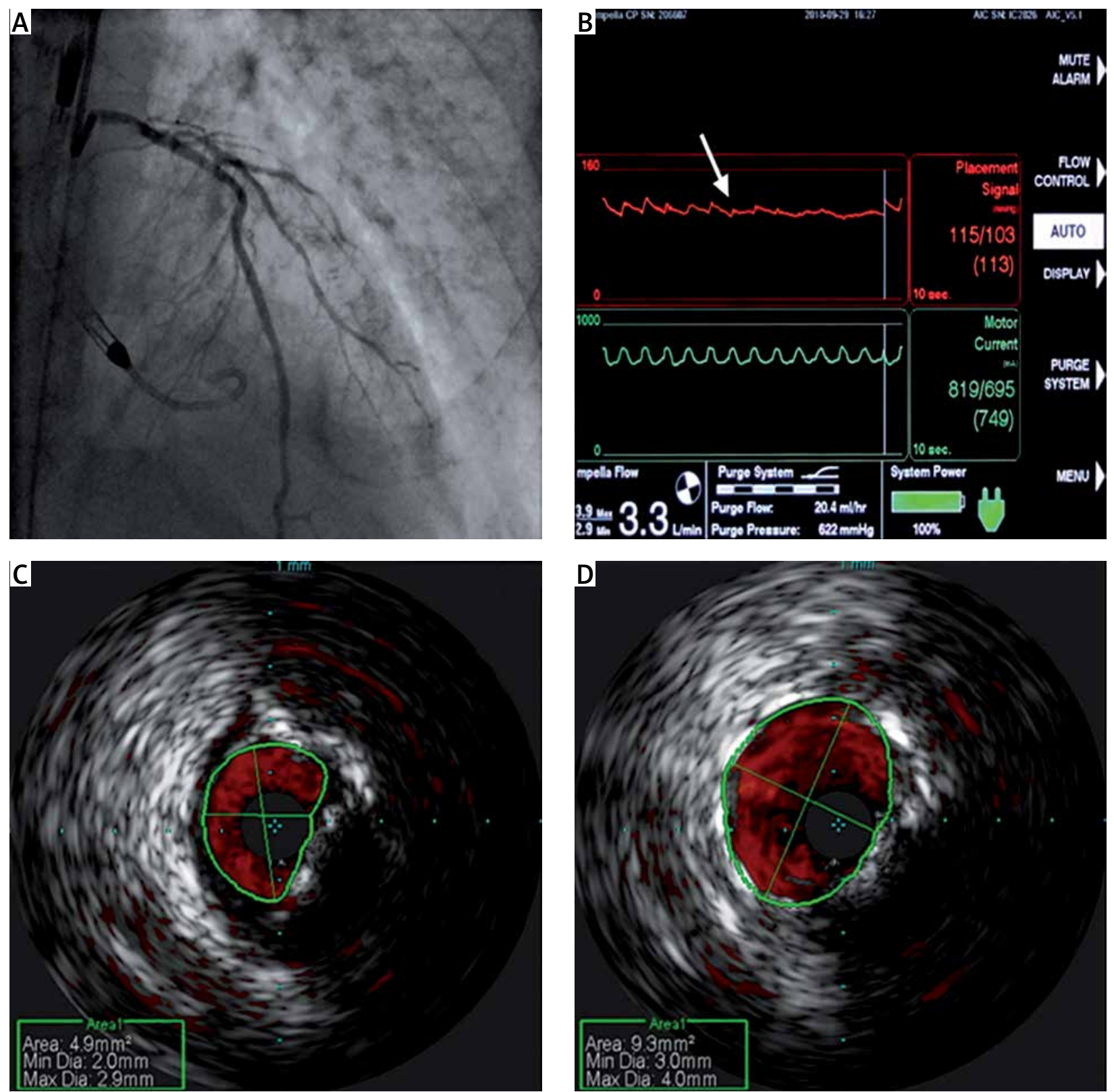

Figure 1. A - Baseline coronary angiography of the left coronary artery: heavy calcifications, occlusion of the circumflex artery and significant stenosis in the distal part of the left main artery (LM) and proximal segment of the left descending artery, B - blood pressure curve straightening after balloon inflation in the LM (arrow), C - significant stenosis of the LM imaged in intravascular coronary ultrasound (ICUS), D - ICUS confirming an optimal stent apposition

\section{References}

1. Al-Rashid F, Hildebrandt HA, Kahlert P. Protected percutaneous coronary intervention - a new road for success in treating complex patients. Adv Interv Cardiol 2016; 1245: 200-2.

2. Shishehbor MH, Moazami N, Tong MZ, et al. Cardiogenic shock: from ECMO to Impella and beyond. Cleve Clin J Med 2017; 84: 287-95.

3. Dudek D, Rakowski T, Sukiennik A, et al. Circulatory support with Impella CP device during high-risk percutaneous coronary interventions: initial experience in Poland. Adv Interv Cardiol 2016; 12: 254-7. 